\title{
Ranibizumab Versus Dexamethasone Implant in Macular Edema Secondary to Ischemic Retinal Vein Occlusion in a Real-Life Practice: A Retrospective Case Control Study
}

\author{
Abdullah Ozkaya*, Hatice Nur Tarakcioglu, Ipek Tanir and Beril Tulu \\ Beyoglu Eye Training and Research Hospital, Istanbul, Turkey

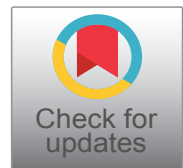

*Corresponding author: Abdullah Ozkaya, Beyoglu Eye Training and Research Hospital, Bereketzade Cami Sok., 34421 Beyoglu, Istanbul, Turkey, Tel:0090-212-251-59-00, E-mail: abdozkaya@gmail.com

\begin{abstract}
Purpose: The aim of this study was to compare the real-life outcomes of Intravitreal Ranibizumab (IVR) and Intravitreal Dexamethasone Implant (IDI) treatments in patients with Macular Edema (ME) secondary to Ischemic Retinal Vein Occlusion (IRVO).

Methods: Retrospective, interventional case control study. The treatment naïve IRVO patients with ME who were treated with Intravitreal Ranibizumab (IVR) or Intravitreal Dexamethasone Implant (IDI) and had a minimum follow-up time of 12 months were included. Primary outcome measure was the change in Best Corrected Visual Acuity (BCVA). Secondary outcome measures were change in central retinal thickness and mean number of injections.

Results: A total of 41 eyes of 41 patients were included. Nineteen patients $(46.3 \%)$ were treated with IVR, 22 patients $(53.7 \%)$ were treated with IDI. Mean BCVA in IVR group at baseline and month 12 was $1.25 \pm 0.58$ and 0.82 \pm 0.54 LogMAR, respectively. Mean BCVA in IDI group at baseline and month 12 was $1.15 \pm 0.52$ and $1.09 \pm 0.54$ LogMAR, respectively. The change in mean BCVA was significantly better in IVR group than IDI group at month 12 ( $p$ $=0.05$ for month $3, p=0.1$ for month $6, p=0.07$ for month 9 , and $p=0.03$ for month 12 , respectively). Mean number of visits during the first year was $5.0 \pm 1.0$ (range 2-6) in IVR group, and $4.0 \pm 0.9$ (range 3-6) in IDI group ( $p=0.004$ ). Mean number of injections was 3.7 \pm 1.2 (range $3-6$ ) in IVR group, and $1.50 \pm 0.6$ (range 1-3) in IDI group ( $p<0.0001)$.

Conclusion: There was a statistically significant difference in regard to change in VA at month 12 between IVR and IDI treatments and the in-group VA changes was statistically better in IVR group which might suggest that IVR might be a better option in ME secondary to IRVO.
\end{abstract}

\section{Keywords}

Dexamethasone, Ischemia, Ranibizumab, Retinal vein occlusion

\section{Introduction}

Around one fifth of the Central Retinal Vein Occlusions (CRVO) and a significant amount of Branch Retinal Vein Occlusions (BRVO) are classified as ischemic via Fluorescein Angiography (FA), and characterized by a reduced perfusion of the retina [1-3]. Central retinal vein occlusions are classified as ischemic CRVO when a capillary non-perfusion area is $>10$-disc areas and BRVOs are classified as ischemic BRVO when a capillary non-perfusion area is > 5-disc areas, detected in fluorescein angiography according to previous studies [1-3]. Most important complications of ischemic Retinal Vein Occlusions (RVO) are Macular Edema (ME) and ocular neovascularizations [3]. Ocular neovascularizations secondary to ischemic RVOs are conventionally treated with panretinal laser photocoagulation for CRVO, and scatter laser photocoagulation for BRVO [3]. However, the treatment options for ME edema secondary to ischemic RVO are still a debate. Focal laser photocoagulation, some surgical approaches, and intravitreal injections of steroids and anti-Vascular Endothelial Growth Factors (anti-VEGF) are the treatments evaluated for ME secondary to RVO [4-15]. However, pivotal multicenter studies regarding about the efficacy of intravitreal injections of steroids and anti-VEGF agents in ME

Citation: Ozkaya A, Tarakcioglu HN, Tanir I, Tulu B (2017) Ranibizumab Versus Dexamethasone Implant in Macular Edema Secondary to Ischemic Retinal Vein Occlusion in a Real-Life Practice: A Retrospective Case Control Study. Int J Ophthalmol Clin Res 4:080. doi.org/10.23937/2378-346X/1410080 Received: June 05, 2017: Accepted: November 01, 2017: Published: November 03, 2017

Copyright: (C) 2017 Ozkaya A, et al. This is an open-access article distributed under the terms of the Creative Commons Attribution License, which permits unrestricted use, distribution, and reproduction in any medium, provided the original author and source are credited. 
secondary to RVO usually excluded the patients with ischemic RVO $[14,15]$. Soon after these studies, some authors evaluated the efficacy of intravitreal treatment in the ischemic subgroup of RVO patients [2,5,7-13]. In this case control study, we aimed to compare the real-life outcomes of Intravitreal Ranibizumab (IVR) and Intravitreal Dexamethasone Implant (IDI) treatments in patients with ME secondary to RVO.

\section{Methods}

Medical records of patients who had ME secondary to IRVO, and underwent IVR or IDI treatment between January 2014 and December 2015 were reviewed in this retrospective case-control study. Treatment naive IRVO patients who had a macular edema $<3$ months at first admission, had a follow-up time at least 12 months were included. Patients who had another retinal disease (e.g., diabetic retinopathy, vitreomacular surface disorder), or non-ischemic RVO were not included. Written informed consent for treatment was obtained from all of the patients, and the study adhered to the tenets of Declaration of Helsinki.

Data collected from patients' records included, age, gender, Best Corrected Visual Acuity (BCVA), and Central Retinal Thickness (CRT) at baseline, month 3, 6, 9, and 12 , and number of visits and injections.

All patients underwent standardized examination, including measurement of BCVA using a projection chart at 4 meters, slit-lamp biomicroscopy and fundus examination, and measurement of Intraocular Pressure (IOP) via applanation tonometry. Fundus photography, fluorescein angiography (HRA-2; Heidelberg Engineering, Heidelberg, Germany), and Optical Coherence tomography (OCT) imaging (Spectralis; Heidelberg Engineering, Heidelberg, Germany) were performed before treatment. All examinations were repeated at all visits, with exception of fluorescein angiography, which was repeated only when cause of VA deterioration could not be clarified in clinical examination or with other imaging methods. OCT was used to measure CRT, which was defined as mean thickness of the neurosensory retina in central $1 \mathrm{~mm}$ diameter region, and was computed via OCT mapping software provided with device. Fluorescein angiography was inspected for capillary dropout zones at the fovea and peripheral retina, and for leakage, which were accepted as causes of ME. Type of disease was defined as ischemic RVO if ischemic area was $\geq$ 5-disc areas in branch retinal vein occlusion patients, or $\geq 10$-disc areas in central retinal vein occlusion patients [1-3].

All injections were performed under sterile conditions after application of topical anesthesia, 10\% povidone-iodine (Betadine; Purdue Pharma, Stamford, CT, USA) scrub was used on the eyelids and eyelashes, and $5 \%$ povidone-iodine was administered to the conjunctival sac. Intravitreal ranibizumab $0.5 \mathrm{mg} / 0.5 \mathrm{ml}$ (Lucen- tis; Novartis, Basel, Switzerland) with a 30 -gauge needle, or IDI $(700 \mu \mathrm{g})$ was injected through the pars plana $3.5 \mathrm{~mm}$ posterior to the limbus with 22-gauge preloaded injection system. Patients were called for infection control 2 days after injection and instructed to return to the hospital if they experienced decreased vision, eye pain, or any new symptoms.

In IVR group initially, some of the patients received a loading dose of three consecutive monthly IVR injections. There were not any strict criteria for giving a loading dose to the patients. Then the patients were followed monthly, and a single injection of IVR was repeated when the VA decreased by one or more lines from the last visit, or any increase in CRT in OCT images. In IDI group, initially, all of the patients received single dose IDI injection. Patients were then followed monthly or bimonthly, and single injection of IDI was repeated when VA decreased by 1 or more lines from previous visit, or when an increase of $>150$ micrometer in CRT was observed in OCT images. Panretinal or scatter laser photocoagulation was performed for the ischemic CRVO and BRVO patients, if new ocular neovascularization was detected at the follow-up visits.

Primary outcome measure of this study was change in BCVA. Secondary outcome measures were change in CRT, number of visits and given injections.

\section{Statistical analysis}

Visual acuity was converted to the Logarithm of the Minimum Angle of Resolution (LogMAR) for statistical analysis. Categorical variables were presented as numbers and percentages, while numerical variables were expressed as mean and standard deviation. First data were analyzed in terms of normality using Shapiro-Wilk test. As the distribution of the data was found to be normal, independent sample t-test was used to compare numerical variables between the 2 groups. Change in BCVA and CRT within the 2 groups were assessed with paired samples t-test. Categorical variables were compared by using chi-square test. $P$ value $<0.05$ was considered statistically significant.

\section{Results}

A total of 41 eyes of 41 patients were included. Nineteen patients $(46.3 \%)$ were treated with IVR, 22 patients (53.7\%) were treated with IDI. The baseline characteristics of the 2 groups were summarized in Table 1.

Mean BCVA in IVR group at baseline, month 3, 6, 9, and 12 was $1.25 \pm 0.58$ LogMAR (range 0.4-2.0), 0.91 \pm 0.59 LogMAR (range 0.0-2.0), $0.81 \pm 0.64$ LogMAR (range 0.1-2.0), $0.89 \pm 0.76$ LogMAR (range 0.1-3.0), and $0.82 \pm 0.54$ LogMAR (range 0.1-2.0), respectively (Figure 1). Mean BCVA in IDI group at baseline, month 3, 6, 9, and 12 was $1.15 \pm 0.52$ LogMAR (range 0.3-2.0), 1.09 \pm 0.57 LogMAR (range 0.15-2.0), $0.98 \pm 0.64$ LogMAR (range 0.15-2.0), $1.07 \pm 0.64$ LogMAR (range 0.15-2.0), 
Table 1: General characteristics of the patients.

\begin{tabular}{|l|l|l|l|}
\hline & IVR & IDI & p value \\
\hline Number of eyes & 19 & 22 & - \\
\hline Age (years) & $58.5 \pm 11.2$ & $63.7 \pm 11.0$ & 0.1 \\
\hline Gender (female/male) & $11 / 8$ & $12 / 10$ & 0.8 \\
\hline Hypertension (\%) & $13(68 \%)$ & $12(54 \%)$ & 0.2 \\
\hline Diabetes (\%) & $4(21 \%)$ & $6(27.2 \%)$ & 0.6 \\
\hline Hyperlipidemia (\%) & $2(7.4 \%)$ & $2(9.0 \%)$ & 0.8 \\
\hline $\begin{array}{l}\text { Macular ischemia on FA } \\
\text { (non-ischemic/ischemic) }\end{array}$ & $4 / 15$ & $8 / 14$ & 0.2 \\
\hline Type of RVO (BRVO/CRVO) & $13 / 6$ & $12 / 10$ & 0.3 \\
\hline Lens status (phakic/pseudophakic) & $17 / 2$ & $13 / 9$ & $0.02^{*}$ \\
\hline Baseline BCVA (LogMAR) & $1.25 \pm 0.58$ & $1.15 \pm 0.51$ & 0.7 \\
\hline Baseline CRT (micrometers) & $640 \pm 191$ & $623 \pm 99$ & 0.5 \\
\hline
\end{tabular}

IVR: Intravitreal Ranibizumab; IDI: Intravitreal Dexamethasone Implant; P: p value; FA: Fluorescein Angiography; BRVO: Branch Retinal Vein Occlusion; CRVO: Central Retinal Vein Occlusion; BCVA: Best Corrected Visual Acuity; LogMAR: Logarithm of the Minimum Angle of Resolution; CRT: Central Retinal Thickness.

* $p$ value less than 0.05 was considered statistically significant.

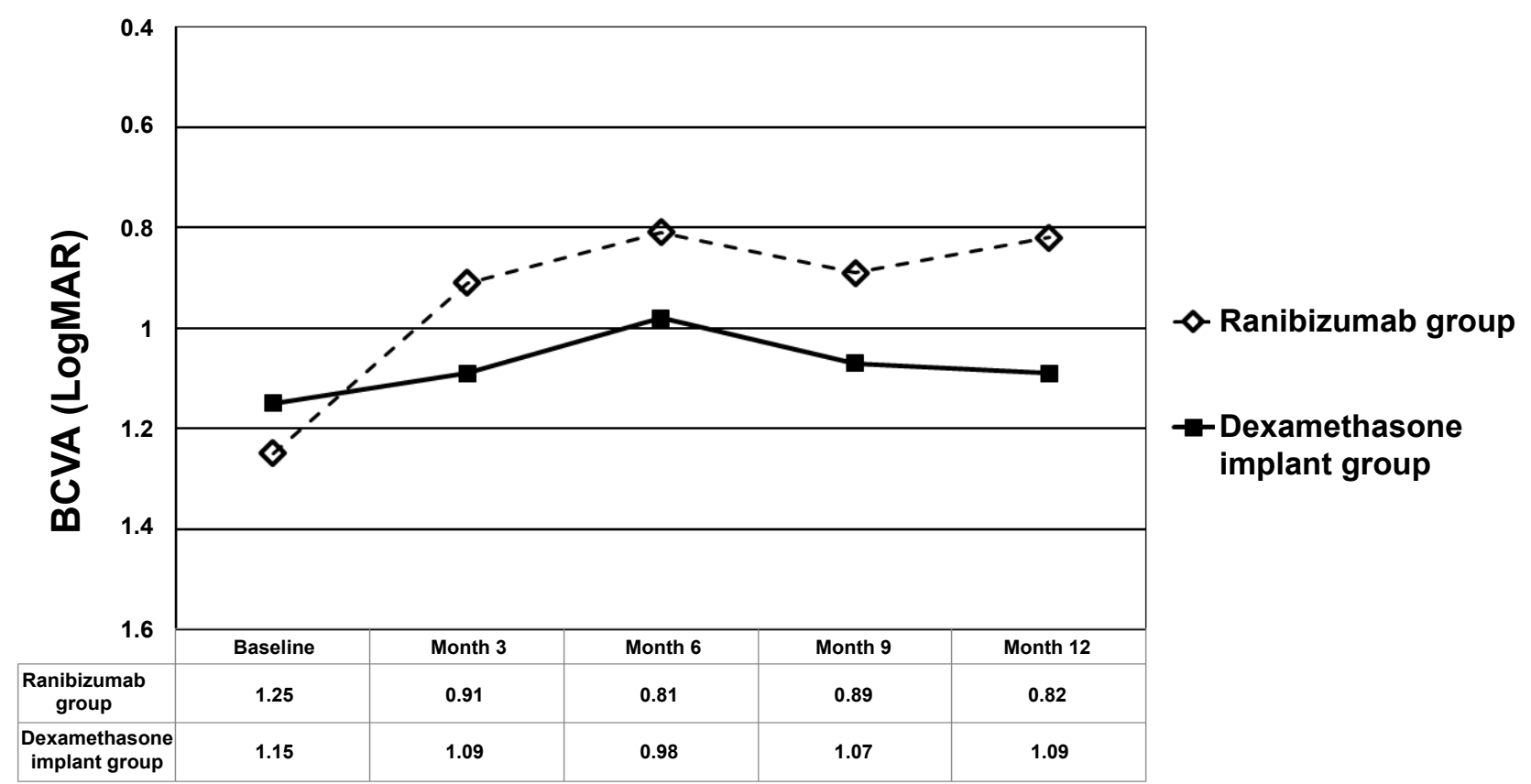

Figure 1: The changes in mean Best Corrected Visual Acuity (BCVA) in ranibizumab and dexamethasone implant groups. The graph shows the mean Logarithm of the Minimum Angle of Resolution (LogMAR) BCVA levels from baseline to month 12.

and $1.09 \pm 0.54$ LogMAR (range 0.15-2.0), respectively (Figure 1). Mean BCVA from baseline to month 3, 6, 9, and 12 was all statistically better in IVR group $(p=0.02$ for month $3, p=0.01$ for month $6, p=0.03$ for month 9 , and $p=0.02$ for month 12, respectively). Mean BCVA from baseline to month $3,6,9$, and 12 was not statistically different in IDI group ( $p>0.05$ for all time points). The change in mean BCVA was significantly better in IVR group than IDI group at month 12 ( $p=0.05$ for month 3 , $p=0.1$ for month $6, p=0.07$ for month 9 , and $p=0.03$ for month 12, respectively). At month 12,8 of the 19 patients (42.1\%) in IVR group, and 5 of the 22 patients $(22.7 \%)$ in IDI group gained VA $\geq 3$ lines $(p=0.3)$. Sixteen of the 19 patients (84.2\%) in IVR group and 19 of the 22 patients $(86.4 \%)$ in IDI group had a stable (lost < 3 lines, stable, or gained $<3$ lines) VA at month $12(p=0.3)$. Only 3 patients in IVR and 3 patients in IDI group lost $\geq 3$ lines of VA at month $12(p=0.3)$

Mean CRT in IVR group at baseline, month 3, 6, 9, and 12 was $640 \pm 191$ micrometers (range 336-894), $483 \pm 167$ micrometers (range 246-874), $371 \pm 168 \mathrm{mi}-$ crometers (range 221-755), $445 \pm 294$ microns (range 236-1147), and $357 \pm 109$ micrometers (range 242-655), respectively (Figure 2). Mean CRT in IDI group at baseline, month $3,6,9$, and 12 was $623 \pm 99$ micrometers (range 457-824), $445 \pm 166$ micrometers (range 226876), $453 \pm 135$ micrometers (range 234-682), $408 \pm 119$ microns (range 207-635), and $460 \pm 194$ micrometers (range 150-987), respectively (Figure 2). Mean CRT from baseline to all time points were found to be significantly decreased in IVR group except for month 3 ( $p=0.1$ for month $3, p=0.001$ for month $6, p=0.01$ for month 9, and $p<0.0001$ for month 12 , respectively). Mean CRT from baseline to all time points were found to be signifi- 


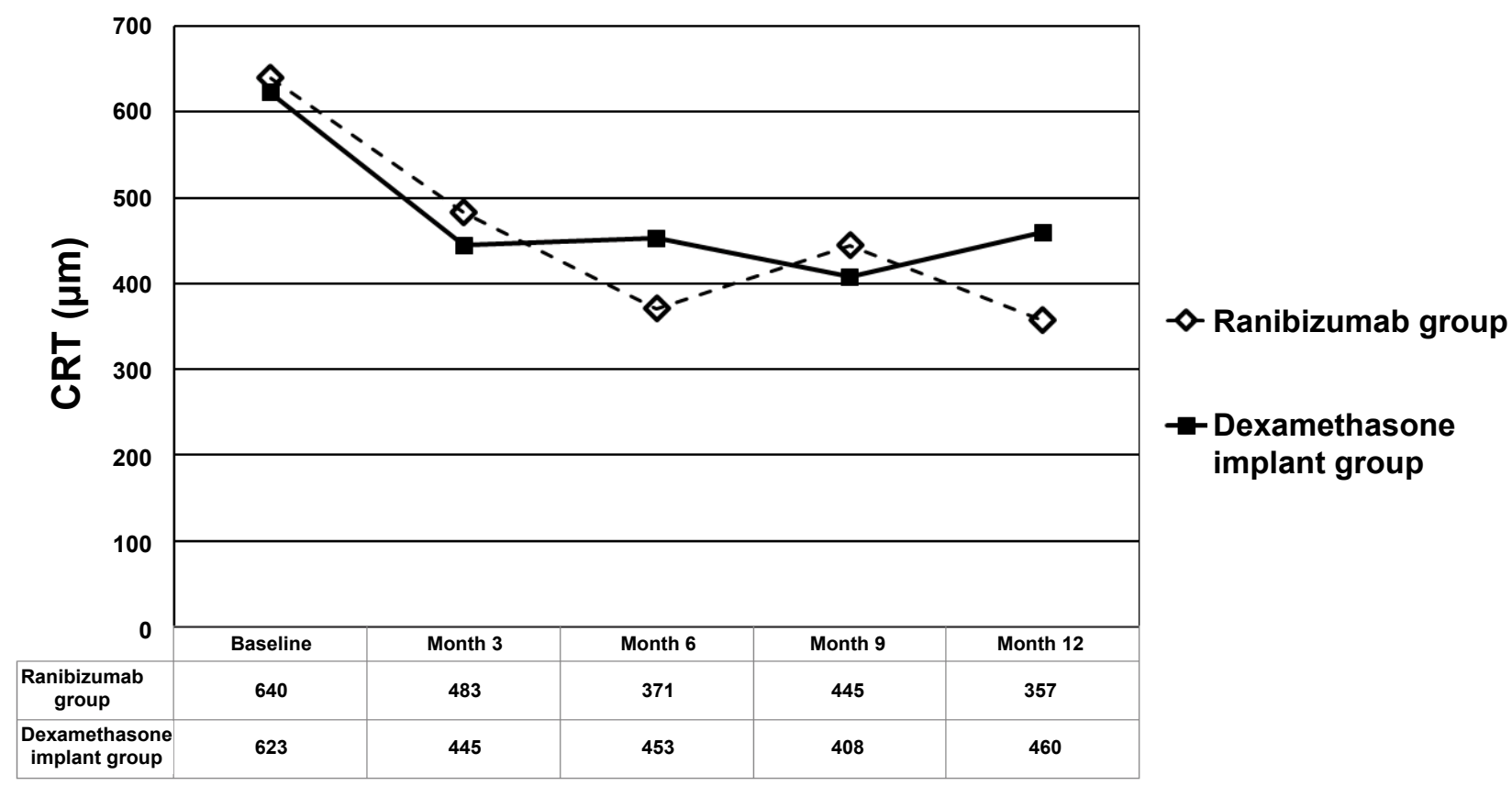

Figure 2: The changes in mean Central Retinal Thickness (CRT) in ranibizumab and dexamethasone implant groups. The graph shows the mean logarithm of the minimum angle of resolution CRT levels from baseline to month 12.

cantly decreased in IDI group $(p<0.0001$ for month 3 , $p=0.001$ for month $6, p<0.0001$ for month 9 , and $p=$ 0.002 for month 12 , respectively). The change in mean CRT was not statistically different between the 2 groups at any of the time points ( $p>0.05$ for all). At month 12, 9 patients $(47.4 \%)$ in IVR group, and 7 patients $(31.8 \%)$ in IDI group had CRT $<350$ micrometers $(p=0.3)$.

Mean number of visits during the first year was 5.0 \pm 1.0 (range 2-6) in IVR group, and $4.0 \pm 0.9$ (range 3-6) in IDI group ( $p=0.004)$. Mean number of injections was $3.7 \pm 1.2$ (range $3-6$ ) in IVR group, and $1.50 \pm 0.6$ (range $1-3)$ in IDI group $(p<0.0001)$.

Seventeen patients (89.5\%) were phakic and 2 (10.5\%) were pseudophakic in IVR group, and 13 patients (68.2\%) were phakic and 7 (31.8\%) were pseudophakic in IDI group $(p=0.03)$. None of the patients in IVR group and seven of the $13(53.8 \%)$ patients in IDI group showed progression in lens opacity $(p<0.0001)$. However only one patient required cataract surgery during the 12 months of follow-up period.

Eleven of the 16 (68.8\%) of ischemic CRVO patients and 11 of 24 (56.0\%) of ischemic BRVO patients required panretinal or scatter laser photocoagulation during the follow-up because of ocular neovascularization, and none of the patients developed neovascular glaucoma during the follow-up period.

None of the patients in IVR group and 6 of 22 patients $(27.3 \%)$ in IDI group indicated increase $>10 \mathrm{mmHg}$ in IOP; however, IOP increase was transient in all patients and treated with only topical antiglaucomatous drops. None of the patients required incisional surgery. No injection-related endophthalmitis was noted after total of 104 injections in both of the groups.

\section{Discussion}

In this case control study, we evaluated the outcomes of IVR and IDI treatments in the subgroup of ischemic RVO patients associated with ME. Mean BCVA from baseline to month 12 was statistically better at all time points in IVR group, in contrast no statistical difference was found in IDI group. Ranibizumab group showed significant visual gain in in-group BCVA changes; however, there was a significant difference between the two groups in regard to visual changes at only month 12 throughout the first year. Also, the percentage of the patients who showed $\geq 3$ lines of VA gain, $\geq 3$ lines of VA loss, or were stable at month 12 were similar between the two groups. Mean CRT was found to be decreased from 640 micrometers to 357 micrometers in IVR group, and from 623 micrometers to 460 micrometers in IDI group. The change in mean CRT was statistically different for both of the groups at all of the time points, except for month 3 in IVR group. The change in mean CRT was not statistically different between the two groups at any of the time points. The mean number of visits and injections of IVR group were both higher than the IDI group as expected. However, they were lower than the prospective randomized controlled studies which reflected the real-life nature of our study. As it is a fact that PRN (Pro re nata) treatment regimen is very difficult to obey in real life in both IVR and IDI treatments, we had planned to perform monthly visits for IVR group and at least bimonthly visits for IDI group; however, the visit number was found to be 5 for IVR group and 4 for IDI group which were much lower than they had to be [6]. Only one of the 22 patients required cataract surgery during the follow-up and 6 of the 22 patients showed transient IOP increase in IDI group. Around two 
thirds of the ischemic CRVO and half of the ischemic BRVO patients showed ocular neovascularization and required laser photocoagulation treatment and none of these patients developed neovascular glaucoma.

In a study by Parodi, et al. the outcomes of IDI treatment over a follow-up time of 12 months were evaluated in 29 patients with ischemic RVO secondary to ME, prospectively [2]. The median visual acuity of BRVO and CRVO patients improved from 48 letters to 62 letters and 10 letters to 37 letters from baseline to month 12, respectively, which are both equal to around 3 lines of visual gain with a mean injection number of 2.8. The increase in visual acuity was dramatically better than our study which might be secondary to prospective design, strict re-treatment criteria and follow-up criteria of the study. In the study, the patients were called for monthly follow-up visits after month 4 and were given an injection on a more proper re-treatment regimen than other study. However, the percentage of the patients who had stable or improved visual acuity at month 12 was similar to our study ( $87 \%$ for CRVO and $92 \%$ for BRVO). The median CRT was found to be decreased from 749 to 363 micrometers in ischemic CRVO group, and from 459 to 323 microns in ischemic BRVO group, respectively from baseline to month 12 . Ocular neovascularization was detected in 7 of the 29 patients at the baseline, and all were reported to be regressed after treatment with laser photocoagulation. As complications of intravitreal steroid use, it was reported that $15 \%$ of the patients showed cataract progression, $26 \%$ of the CRVO patients and $21 \%$ of the BRVO patients required topical antiglaucomatous treatment which were similar to our study. Chatziralli, et al. studied the long-term effect of anti-VEGFs in patients with ischemic CRVO [7]. They included 15 eyes of 15 patients retrospectively, and evaluated the outcome of anti-VEGF treatment over a follow-up time of 24 months. The mean number of anti-VEGF injections throughout the first and second year was reported to be 7.4 and 3.5 , respectively, which were higher than our study. A total of the $64.3 \%$ of the patients showed resolution of ME at month 24 . The maximum VA gain was reported as 10.7 letters at month 12 in the study. Although the mean injection number was very low in our study the visual acuity changes at month 12 was similar, as our patients showed a mean of 4.3 lines of VA gain at month 12 . This might be because of the worse mean baseline BCVA of our study population as it is a known fact from the previous studies that patients with worse VA levels tend to gain more vision (51 letters in Chatziralli study approximately is equal to 0.9 LogMAR vs. 1.25 LogMAR in our study) [7]. Mean baseline CRT was reported to be 593 micrometers in the study, and it was reported to decrease to 351 micrometers at month 12 and 349 micrometers at month 24 . The baseline CRT of the IVR group in our study was 640 microns and decreased to 357 micrometers at month 12 which was a similar outcome.
The main limitation of this study was the retrospective design and both BRVO and CRVO patients were evaluated together because of low patient number. Also, some participants of the IVR group received 3 loading doses whereas others did not. However, the study included only treatment naive patients and to the best of our knowledge this was the first case-control study in the literature which allowed us to compare IVR and IDI treatments in a real-life practice in patients with ME secondary ischemic RVO patients.

In conclusion, there was a statistically significant difference in regard to change in VA at month 12 between IVR and IDI treatments and the in-group VA changes were statistically better in IVR group which might suggest that IVR might be a better option in ME secondary to ischemic RVO.

\section{Disclosure}

The authors report no conflicts of interest in this work.

\section{Summary}

In this study we compared the real-life treatment outcomes of ranibizumab and dexamethasone implant in macular edema secondary to ischemic retinal vein occlusions. This is the first study comparing the two drugs in this subgroup, and ranibizumab seemed more effective than dexamethasone implant in regard to visual outcomes.

\section{References}

1. The Central Vein Occlusion Study Group (1997) Natural history and clinical management of central vein occlusion. Arch Ophthalmol 115: 486-491.

2. Parodi $M B$, lacono $P$, Petruzzi $G$, Parravano $M$, Varano $M$, et al. (2015) Dexamethasone implant for macular edema secondary to ischemic retinal vein occlusions. Retina 35 : 1387-1392.

3. Hayreh SS, Zimmerman MB, Podhajsky P (1994) Incidence of various types of retinal vein occlusion and their recurrence and demographic charecteristics. Am J Ophthalmol 117: 429-441.

4. Ozkaya A, Celik U, Alkin Z, Faiz Turan M, Yazici AT, et al. (2013) Comparison between intravitreal triamcinolone with grid laser photocoagulation versus bevacizumab with grid laser photocoagulation for branch retinal vein occlusion. ISRN Ophthalmol 2013: 141279.

5. Tam EK, Golchet P, Yung M, DeCroos FC, Spirn M, et al. (2017) Ischemic central retinal vein occlusion in the anti-vascular endothelial growth factor era. Retina.

6. Ozkaya A, Garip R, Yesilkaya C, Alkin Z, Taskapili M (2017) Ranibizumab versus aflibercept in macular edema secondary to retinal vein occlusion in real life; a retrospective case control study. Beyoglu Eye J 1: 13-18.

7. Chatziralli I, Theodossiadis G, Parikakis E, Mitropoulos PG, Theodossiadis P (2017) Long-term anatomical and functional outcomes in patients with ischemic central retinal vein occlusion treated with anti-vascular endothelial growth factor agents. Ophthalmic Res 58: 203-208. 
8. Bradshaw SE, Gala S, Nanavaty M, Shah A, Mwamburi M, et al. (2016) Systematic literature review of treatments for management of complications of ischemic central retinal vein occlusion. BMC Ophthalmol 16: 104.

9. Shirakata $Y$, Fujita T, Nakano $Y$, Shiraga F, Tsujikawa A (2016) Pars plana vitrectomy combined with internal limiting membrane peeling to treat persistent macular edema after anti-vascular endothelial growth factor treatment in cases of ischemic central retinal vein occlusion. Case Rep Ophthalmol 7: 1-8.

10. Asano S, Miyake K, Miyake S, Ota I (2007) Relationship between blood-aqueous barrier disruption and ischemic macular edema in patients with branch or central retinal vein occlusion: effects of sub-tenon triamcinolone acetonide injection. J Ocul Pharmacol Ther 23: 577-584.

11. Feltgen N, Junker B, Agostini H, Hansen LL (2007) Retinal endovascular lysis in ischemic central retinal vein occlusion: one-year results of a pilot study. Ophthalmology 114 716-723.
12. Mirshahi A, Roohipoor R, Lashay A, Mohammadi SF, Mansouri MR (2005) Surgical induction of chorioretinal venous anastomosis in ischaemic central retinal vein occlusion: a non-randomised controlled clinical trial. $\mathrm{Br} \mathrm{J}$ Ophthalmol 89: 64-69.

13. Brown DM, Wykoff CC, Wong TP, Mariani AF, Croft DE, et al. (2014) Ranibizumab in preproliferative (ischemic) central retinal vein occlusion: the rubeosis anti-VEGF (RAVE) trial. Retina 34: 1728-1735.

14. Campochiaro PA, Brown DM, Awh CC, Lee SY, Gray S, et al. (2011) Sustained benefits from ranibizumab for macular edema following central retinal vein occlusion: twelvemonth outcomes of a phase III study. Ophthalmology 118: 2041-2049.

15. Heier JS, Campochiaro PA, Yau L, Li Z, Saroj N, et al. (2012) Ranibizumab for macular edema due to retinal vein occlusions: long-term follow-up in the HORIZON trial. Ophthalmology 119: 802-809. 